\title{
Квантово-оптические технологии для обработки и передачи информации
}

\author{
А.К. Федоров \\ Российский квантовый иенттр \\ Московский физико-технический институт \\ E-mail: $\underline{\text { akf@rqc.ru }}$
}

DOI: $10.31868 /$ RFL2020.157

Сегодня квантовые технологии - одно из наиболее бурно развивающихся направлений. Квантовые технологии открывают новые возможности для целого ряда областей. За счет своих уникальных свойств, квантовые системы могут стать основой нового поколения высокопроизводительных вычислительных устройств (квантовых компьютеров), методов защиты информации (с использованием квантовой криптографии), а также высокоточных измерительных устройств (квантовых сенсоров и квантовых метрологических устройств).

Прогресс последнего времени связан с разработкой и внедрением квантовооптических технологий для обработки и передачи информации. В частности, активно развивается направление квантового распределения ключей - возможности использовать передачу одиночных квантовых состояний света для конфиденциального обмена ключами между удаленными пользователями. В докладе будет представлен обзор экспериментов по квантовому распределению ключей в городских условиях $[1,2]$.

Квантово-оптические технологии также активно развиваются в контексте технологии квантовых вычислений. Во-первых, как метод управления квантовыми системами, использующими ультрахолодные атомы и ионы. Во-вторых, как самостоятельная вычислительная платформа. В докладе будет представлен анализ ряда достижений в области создания квантовых вычислительных систем, использующих квантово-оптические технологии в качестве основного метода экспериментального контроля [3]. В частности, будут представлены предварительные результаты, полученные в рамках проекта по созданию квантового вычислительного устройства на основе ионов.

Работа поддержана грантом МК-923.2019.2.

\section{Литература}

[1] A.V. Duplinskiy, E.O. Kiktenko et al, J. Russ. Laser Res. 39, 113 (2018).

[2] E.O. Kiktenko, N.O. Pozhar et al, Quant. Sci. Technol. 3, 035004 (2018).

[3] E.O. Kiktenko, A.S. Nikolaeva et al, Phys. Rev. A 101, 022304 (2020). 